\title{
On the location of the supermassive black hole in CTA 102
}

\author{
Christian M. Fromm ${ }^{1}$, Manel Perucho ${ }^{2,3}$, Eduardo Ros ${ }^{1,2,3}$, Tuomas Savolainen ${ }^{1,4}$, and J. Anton Zensus ${ }^{1}$ \\ 1 Max-Planck-Insitut für Radioastronomie, Auf dem Hügel 69, 53121 Bonn, Germany \\ e-mail: [cfromm;ros; tukasa; azensus] @mpi fr.de \\ 2 Departament d'Astronomia i Astrofísica, Universitat de València, C/ Dr. Moliner 50, 46100 Burjassot (València), Spain \\ e-mail: Manel.Perucho@uv.es \\ 3 Observatori Astronòmic, Parc Científic, Universitat de València, C/ Catedràtic José Beltrán 2, 46980 Paterna (València), Spain \\ ${ }^{4}$ Aalto University Metsähovi Radio Observatory, Metsähovintie 114, 02540 Kylmälä, Finland
}

Received 11 October 2013 / Accepted 3 December 2014

\begin{abstract}
Context. Relativistic jets in active galactic nuclei are one of the most powerful phenomena in the Universe. They form in the surroundings of the supermassive black holes as a by-product of accretion onto the central black hole in active galaxies. The flow in the jets propagates at velocities close to the speed of light. The distance between the first part of the jet that is visible in radio images (core) and the black hole is still a matter of debate.

Aims. Only very-long-baseline interferometry observations resolve the innermost compact regions of the radio jet. These observations can access the jet base, and by combining data at different wavelenghts, address the physical parameters of the outflow from its emission.

Methods. We have performed an accurate analysis of the frequency-dependent shift of the VLBI core location for a multi-wavelength set of images of the blazar CTA 102 including data from $6 \mathrm{~cm}$ down to $3 \mathrm{~mm}$.

Results. The measure of the position of the central black hole, with mass $\sim 10^{8.93} M_{\odot}$, in the blazar CTA 102 reveals a distance of $\sim 8 \times 10^{4}$ gravitational radii to the $86 \mathrm{GHz}$ core, in agreement with similar measures obtained for other blazars and distant radio galaxies, and in contrast with recent results for the case of nearby radio galaxies, which show distances between the black hole and the radio core that can be two orders of magnitude smaller.
\end{abstract}

Key words. galaxies: active - quasars: individual: CTA102 - radiation mechanisms: non-thermal - radio continuum: galaxies galaxies: jets

\section{Introduction}

The blazar CTA 102 has a redshift $z=1.037$ (Schmidt 1965) a luminosity distance of $6.7 \mathrm{Gpc}$, and an image scale of $8.11 \mathrm{pc} /$ milliarcsec and hosts a supermassive black hole $(\mathrm{SMBH})$ of $10^{8.93} M_{\odot}$ (Zamaninasab et al. 2014). The source was one of the first to show strong variability in the radio after its discovery in the 1960s. Its radio morphology shows a strong core with a southward jet on pc-scales, and two lobes in the northwest and southeast directions (e.g., Stanghellini et al. 1998; Fromm et al. 2013a).

The radio core of a jet is defined as its first observed surface at a given frequency out of the black hole neighborhood. The position at which it appears at each frequency may be different if the core corresponds to the first region that is optically thin to radiation at this frequency, the lower frequencies appearing downstream of the higher ones as the jet flow becomes more dilute and more transparent (Marcaide \& Shapiro 1984; Lobanov 1998). If the core corresponds to a strong reconfinement shock, the core at the highest frequencies should converge at the position of the shock. A recent study of the radio galaxy M 87 (Hada et al. 2011; Marscher 2011) suggested that the core corresponds to the first optically thin surface and that the $43 \mathrm{GHz}$ surface is at 14 gravitational radii $\left(R_{\mathrm{s}}\right)$ from the central black hole. This distance has been suggested to be of $10^{4}-10^{5}$ gravitational radii in the case of blazars (Marscher et al. 2008, 2010) and more distant radio galaxies than M 87 such as 3C 111 and 3C 120 (Marscher et al. 2002; Chatterjee et al. 2009, 2011).
Fundamental differences between radio galaxies and blazar jets have been invoked to explain the difference (Hada et al. 2011; Marscher 2011). In blazars, the jet is observed at a very small angle to the line of sight, which favors the observation of the fast flow due to the relativistic Doppler boosting of the radiation (e.g., Zensus 1997). In radio galaxies, the jet is typically observed at a larger angle, and the faster flow could be missed because of the de-boosting of the radiation. The observed jet in the two types of sources could correspond to the slower, outer layers of the jet, which originated in the accretion disk surrounding the central black hole in the case of radio galaxies, and to the faster spine of the jet generated closer to the black hole, respectively. Therefore, the physical properties of the observed radio core in the two types of objects could be different.

We use $86 \mathrm{GHz}$ VLBA observations made before the start of the flare as observed at $220 \mathrm{GHz}$ (2005.6) Fromm et al. (2011) together with observations down to $5 \mathrm{GHz}$ to measure the distance from the black hole to the radio-core at $86 \mathrm{GHz}$. This observation made during a quiescent state of the jet provides an opportunity to measure the core shift in a clean setting without the potential confusing effects of the flaring component. We expect changes in the opacity of the region during the flare, if it is followed by the propagation of a component, thus changing the measured distances following the core-shift method (Lobanov 1998).

Moreover, Fromm et al. (2013b) showed that the core-shift vectors on the plane of the sky presented a very irregular 


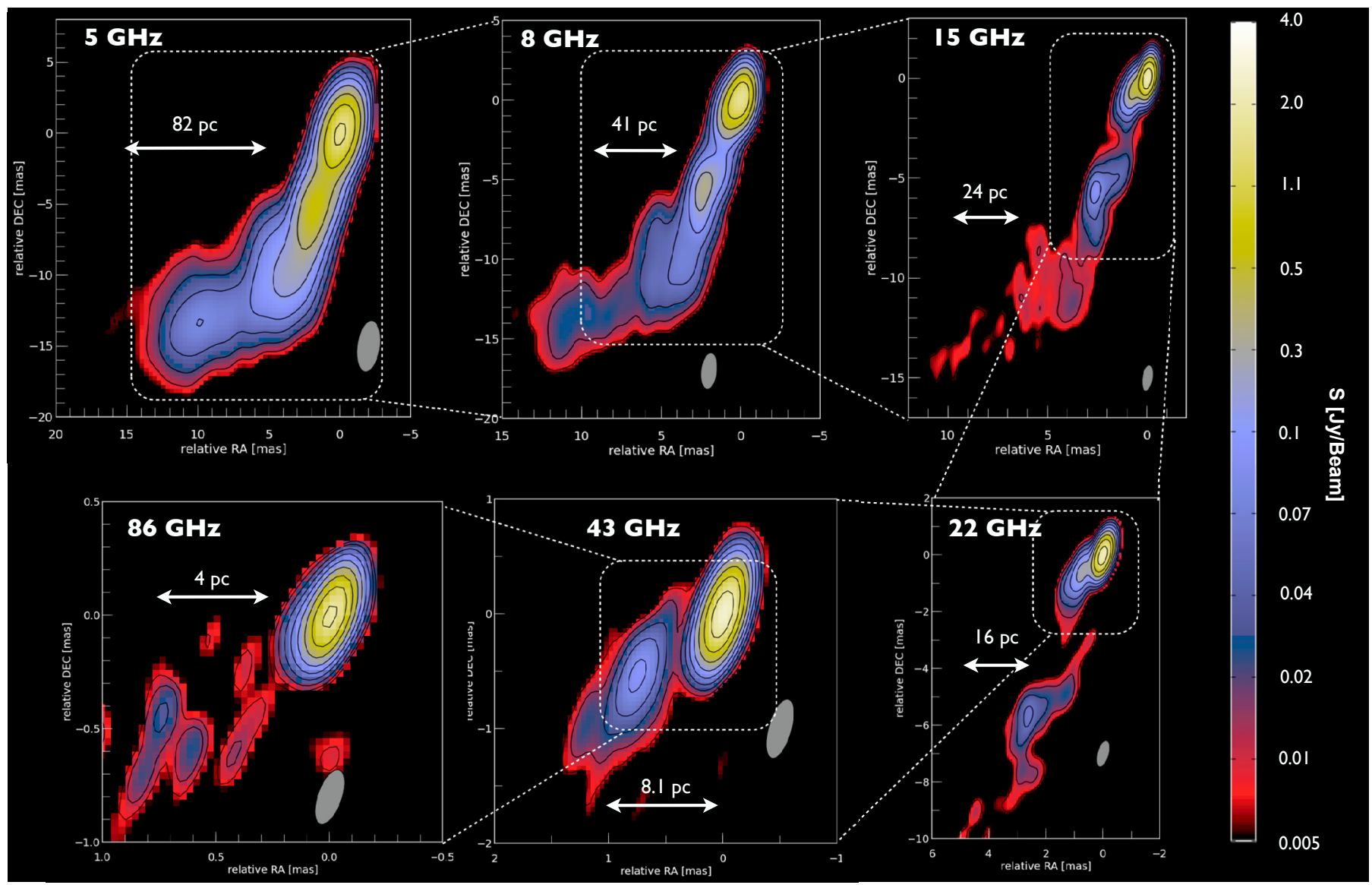

Fig. 1. Parsec-scale radio images of the blazar CTA 102 as observed with the VLBA on 2005-May-19. The interferometric beam is drawn at the bottom-right of each image; the linear scales are also shown. Contours are drawn on the false color image, the lowest one at $0.005 \mathrm{Jy} / \mathrm{beam}$, and are spaced in factors of 2 . At the redshift of $z=1.037$ (luminosity distance of $6.9 \mathrm{Gpc}$ ) 1 mas corresponds to a distance of $8.11 \mathrm{pc}$ and $8.4 \times$ $10^{4}$ gravitational radii for a black hole mass of $10^{10} M_{\odot}$.

behavior during the 2006 flare. The comparison of the steady state with these epochs would require a detailed understanding of the non-radial motions at the core, which is out of the scope of this paper. We refer the reader to recent works that show this behavior, known as jet wobbling, in other sources (Agudo et al. 2007, 2012; Molina et al. 2014). This paper is structured as follows: in Sect. 2 we present the relevant data for this work; in Sect. 3 we present our results; Sect. 4 is devoted to a brief discussion on possible effects that may have an influence on the result; and in Sect. 5 we summarize our work.

\section{VLBA observations and data analysis}

We observed the jet in the blazar CTA 102 using the Very Long Baseline Array (VLBA) at different frequencies, ranging from $5 \mathrm{GHz}$ to $86 \mathrm{GHz}$. The results from these observations were presented in Fromm et al. (2011, 2013a,b), except for the case of the $86 \mathrm{GHz}$ data, which is presented in Fig. 1. These observations cover two years around a major flare in the source in 2006 (Fromm et al. 2011). The $86 \mathrm{GHz}$ epoch that we use here (2005.4) is the only one of the eight observations that yields a high enough signal-to-noise ratio in the extended jet emission to allow alignment of the $86 \mathrm{GHz}$ image with the lower frequencies. In addition, the strong flares observed in many radio sources are usually connected with the later detection of a region of enhanced emission that travels along the jet and can be followed by fitting the interferometric data with a Gaussian function (usually called component) at each epoch. These injected components can be related to an increase in the number of particles injected (Perucho et al. 2008), which can affect the opacity in the region and change the relative position of the core at different frequencies (Kovalev et al. 2008).

Figure 1 shows the core region at all frequencies for epoch 2005.4. We aligned the images of the jet at different frequencies at each epoch using a cross-correlation method based on the optically thin jet regions (Croke \& Gabuzda 2008; Fromm et al. 2013b). This analysis revealed a 2D shift of the core that can only be explained by non-axial (pattern or flow) motion of the emitting region (Fromm et al. 2013b; see also, e.g., Agudo et al. 2007, 2012; Perucho et al. 2012).

Figure 2 shows the total core shifts, computed by integrating the $2 \mathrm{D}$ path through all the intermediate frequencies. Our results for the first epoch show that the core does not converge to zero at our highest frequency, i.e., the measurable shift between the $86 \mathrm{GHz}$ and $43 \mathrm{GHz}$ cores. This result is consistent with the $86 \mathrm{GHz}$ core still corresponding to the $(\tau=1)$ surface.

The fit of the relative position of the core at the different frequencies with respect to the largest $\left(r_{\text {core }} \propto v^{-1 / k_{\mathrm{r}}}\right.$, Lobanov $1998)$ results in a value $\left(k_{\mathrm{r}}=1.0 \pm 0.1\right)$ compatible with the expected in the case of a conical jet in which the energies of the non-thermal particles and the magnetic field are in equipartition, close to the minimum value of those energies required to explain the observed radio flux. This supports the interpretation of the jet in CTA 102 becoming transparent to the different frequencies as it expands. 
Table 1. Core shift values relative to reference (highest-observed) frequency for different epochs.

\begin{tabular}{|c|c|c|c|c|c|c|c|c|c|c|c|}
\hline Epoch & $v_{\text {ref }}$ & $43 \mathrm{G}$ & & $22 \mathrm{GHz}$ & & $15 \mathrm{C}$ & & $8 \mathrm{GHz}$ & & $5 \mathrm{GHz}$ & \\
\hline [yyy & $\mathrm{GHz}]$ & $\begin{array}{c}r \\
{[\mathrm{mas}]}\end{array}$ & $\begin{array}{l}\mathrm{PA} \\
{\left[{ }^{\circ}\right]}\end{array}$ & $\begin{array}{c}r \\
{[\mathrm{mas}]}\end{array}$ & PA & $\begin{array}{c}r \\
{[\mathrm{mas}]}\end{array}$ & $\begin{array}{l}\mathrm{PA} \\
{\left[{ }^{\circ}\right]}\end{array}$ & $\begin{array}{c}r \\
{[\mathrm{mas}]}\end{array}$ & $\begin{array}{l}\mathrm{PA} \\
{\left[{ }^{\circ}\right]}\end{array}$ & $\begin{array}{c}r \\
{[\mathrm{mas}]}\end{array}$ & $\begin{array}{l}\text { PA } \\
{\left[{ }^{\circ}\right]}\end{array}$ \\
\hline 2005-Мay-19 & 86 & $0.03 \pm 0.04$ & 6 & $0.10 \pm 0.06$ & 40 & $0.20 \pm 0.08$ & 103 & $0.32 \pm 0.11$ & 74 & $0.57 \pm 0.16$ & 90 \\
\hline
\end{tabular}

Notes. The uncertainties presented here differ from the ones presented in Fromm et al. (Fromm et al. 2013b). Here we used a more conservative estimate for the uncertainties of the image alignment. The PAs are given in the definition of sky-plane (counter-clockwise from north) not in the mathematical definition as in Fromm et al. (2013b).
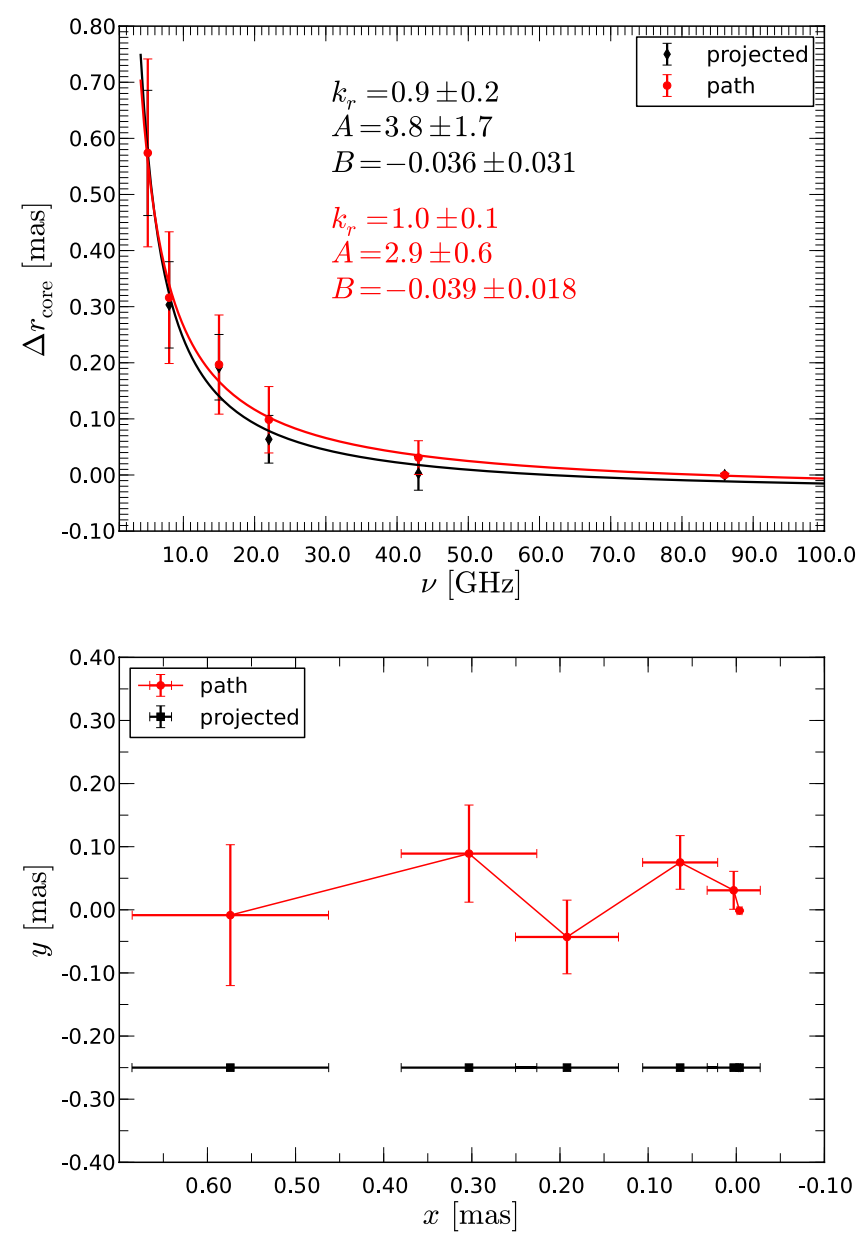

Fig. 2. Top: core shift results as a function of frequency in the jet direction for CTA 102 at epochs 2005.4 (see Table 1). The red points correspond to the core shifts along the $2 \mathrm{D}$ path and the black ones to the core-shifts projected along the average PA of $90^{\circ}$. The solid curves represent a fit to the data with the formula $\Delta r=\mathrm{A} v^{-1 / k_{\mathrm{r}}}+B$ and the values are given in the plot. Bottom: core shift in the sky plane. The red points correspond to the $2 \mathrm{D}$ path and the black points to the projection along the average PA of $90^{\circ}$ shifted in the $y$-direction by -0.25 mas.

The single-dish data from the 2006 radio flare allow us to follow the evolution of the source luminosity with time at different frequencies. The first increase in flux density associated with the flare was detected at a turnover frequency of $222 \pm 99 \mathrm{GHz}$ in 2005.6 (Fromm et al. 2011). Subtracting the spectrum of the source previous to the flare allows us to follow the evolution of the injected flow related to the flare. By doing this, we could identify extra flux at frequencies larger than $100 \mathrm{GHz}$ before the main radio flare, i.e., before the radio feature could be observed out of the radio core at high frequencies. We can assign this extra flux to the injected flux beyond errors and claim that it is optically thin at those frequencies before it is at $43 \mathrm{GHz}$ or $86 \mathrm{GHz}$. This provides independent evidence of the relation of radio-cores to optical depth in this source. Our conclusion is that core-shift analysis performed at higher frequencies than allowed by present techniques would result in non-convergence of the core at a given position. We note that our observations indicate that a standing feature, possibly a re-confinement shock lying 0.1 mas away from the core at $43 \mathrm{GHz}$. This leaves room for the coincidence, within errors, of the core and a re-confinement shock in other sources.

\section{Results: relative location of the black}

Using the results of the power law fit to the obtained core shifts and a viewing angle of $\vartheta=2.6^{\circ}$ (Fromm et al. 2013b), we can derive the distance of the black hole to the radio core at $86 \mathrm{GHz}$ (see Fig. 2). Following the approach of Hada et al. (2011) the distance to the black hole is $(7.0 \pm 3.2) \mathrm{pc}$, which is equivalent to $(8.5 \pm 3.9) \times 10^{4}$ gravitational radii. In order to validate our result we compute the distance to the black hole using the projected core shift along the average PA of $90^{\circ}$ since the calculation along the $2 \mathrm{D}$ path could lead to an overestimation of the distance. The distance obtained using the projected core shifts is $(6.4 \pm 5.5) \mathrm{pc}$ corresponding to $(7.8 \pm 6.7) \times 10^{4}$ gravitational radii similar to the one using the core shifts along the $2 \mathrm{D}$ path.

The distance to the black hole that we obtain fits nicely to the results obtained by Kutkin et al. 2014) and Zamaninasab et al. 2013) for the blazar 3C $454.3\left(r_{\text {core }, 43 \mathrm{GHz}} \sim 9 \mathrm{pc}\right)$. It is also comparable with the predictions for blazar and quasar jets (Marscher et al. 2008, 2010) and distant radiogalaxies (Marscher et al. 2002; Chatterjee et al. 2009, 2011). It is much larger than in the case of the radio galaxy M 87 (Hada et al. 2011; Marscher 2011).

The reason for this difference could be a matter of resolution and that observing M 87 at the distance of those other radiogalaxies would bring the core to $10^{(4-5)} R_{\mathrm{s}}$. In this context, HST-1 (at 1 arcsec from the core) could be observed as the radiocore or within it of M87, with the particularity that it can be identified with a recollimation shock. Another relevant aspect is the viewing angle. Sources observed at small viewing angles pile up all the emission from the compact, bright regions. Therefore, if the jet brightness is high near the radio-core, it may coincide with the last bright, projected surface, which obscures all the regions between the $\tau=1$ surface and this last surface. This effect could again be avoided by increasing resolution.

A relevant limitation of this kind of measure is set by the uncertainties in the alignment of the highest frequency image, especially if no extended emission that aligns properly with lower frequencies is available.

Despite all the difficulties associated with this calculation (see the discussion), we can claim that the separation of the radio core at tens of $\mathrm{GHz}$ to the black hole in CTA 102 is on the order 
of parsecs, implying a separation of $10^{(4-5)} R_{\mathrm{s}}$, on the basis of the values obtained for the $22 \mathrm{GHz}$ (see Table 4 in Fromm et al. $2013 \mathrm{~b}$ ), $43 \mathrm{GHz}$, and $86 \mathrm{GHz}$ radio-cores.

\section{Discussion}

\subsection{Core shifts and the 2006 flare in CTA 102}

It is difficult to derive conclusions from the influence of the passage of the component through the core region because of the large errors obtained in the calculation of the core shifts for the affected epochs. However, a general trend that we observe by performing the same kind of analysis for the remaining epochs is that the exponent of the core shift with frequency decreases when a perturbation crosses the core region (see Table 5 in Fromm et al. 2013b). Actually, the crossing of the $43 \mathrm{GHz}$ core by another component at the beginning of 2007 (Fromm et al. 2013a) seems to cause the same effect as the 2006 flare (see Table 5 in Fromm et al. 2013b). This effect can be assigned to changes in the opacity as the perturbation propagates. As the opacity increases, the high-frequency core positions are dragged downstream, and the relative core shifts are reduced. This is reflected in the decrease of $k_{\mathrm{r}}$.

\subsection{Core shifts and jet wobbling}

Fromm et al. (2013b) showed that the core-shift direction may also change in the plane of the sky. This effect could be due to jet wobbling, which has been observed in other sources (e.g., Agudo et al. 2007, 2012). We would like to point out that these changes in the relative positions of the cores on the plane of the sky could result in changes in the measured projected projected core shifts. In addition, this effect is independent from the presence of a perturbation, although a perturbation can introduce further changes. Both effects seem to be acting on the jet in CTA 102, which makes it very difficult to disentangle their relative roles.

However, even the large apparent changes in the direction of the core shift vectors observed at the first two epochs presented in Fromm et al. (2013b), one during the steady state and the other at the first stages of the evolution of the flare) do not translate into significant changes in the value of $k_{\mathrm{r}}$ obtained for both epochs $(1.0 \pm 0.1$ versus $0.8 \pm 0.3)$.

Nevertheless, the changes of the relative positions on the plane of the sky should have a negligible effect, save errors, on the calculation of the relative location of the black hole, because of the deprojection done to obtain it (Lobanov 1998).

\section{Summary}

We present here the first $86 \mathrm{GHz}$ map of CTA 102 within the series of observations around the 2006 flare. Unfortunately the other monitoring images at this frequency show poor quality. The map presented here corresponds to the first of those epochs, prior to the triggering of the flare. Making use of the simultaneous observations of the source from $5 \mathrm{GHz}$ to $86 \mathrm{GHz}$ and considering that the core is unaffected in this epoch by the propagation of any component, we have measured the core shifts during what we expect to be a steady-state like situation in CTA 102. As a result, we obtain a slope for the core shifts $\left(k_{\mathrm{r}}=1.0 \pm 0.1\right)$ that is compatible with a conical jet in adiabatic expansion. This result allows us to compute the relative position of the $86 \mathrm{GHz}$ core to the black hole, which is $\simeq 7 \mathrm{pc}$ or $8.5 \times 10^{4}$ gravitational radii.

We have discussed the possible effect of perturbations and changes on the directions of the core-shift vector and the estimates of the distance between the radio-core and the black hole. Despite the uncertainties, the same kind of calculations were performed for all epochs using the $22 \mathrm{GHz}$ and $43 \mathrm{GHz}$ cores as reference and values were provided for the distance between them and the black-hole on the order of several parsecs in all cases, confirming our result (Fromm et al. 2013b). Future work should include the validation of our results and possibly confirm the discussed effects on them.

Acknowledgements. We acknowledge L. Fuhrmann for careful reading and for useful comments and suggestions to the manuscript. We thank the anonymous referee for comments and criticism that helped to improve this manuscript. C.M.F. was supported for this research through a stipend from the International Max Planck Research School (IMPRS) for Astronomy and Astrophysics at the Universities of Bonn and Cologne. Part of this work was supported by the COST Action MP0905 "Black Holes in a violent Universe". E.R. acknowledges partial support from MINECO grants AYA2009-13036-C02-02 and AYA2012-38491C02-01 as well as Generalitat Valenciana grant PROMETEO/2009/104. M.P. acknowledges financial support from MINECO grants AYA2010-21322-C03-01, AYA2010-21097-C03-01, and CONSOLIDER2007-00050. This work is based on observations with the VLBA, which is operated by the NRAO, a facility of the NSF under cooperative agreement by Associated Universities Inc. This research made use of data from the MOJAVE database that is maintained by the MOJAVE team (Lister et al. 2009).

\section{References}

Agudo, I., Bach, U., Krichbaum, T. P., et al. 2007, A\&A, 476, L17 Agudo, I., Marscher, A. P., Jorstad, S. G., et al. 2012, ApJ, 747, 63 Chatterjee, R., Marscher, A. P., Jorstad, S. G., et al. 2009, ApJ, 704, 1689 Chatterjee, R., Marscher, A. P., Jorstad, S. G., et al. 2011, ApJ, 734, 43 Croke, S. M., \& Gabuzda, D. C. 2008, MNRAS, 386, 619

Fromm, C. M., Perucho, M., Ros, E., et al. 2011, A\&A, 531, A95

Fromm, C. M., Ros, E., Perucho, M., et al. 2013a, A\&A, 551, A32

Fromm, C. M., Ros, E., Perucho, M., et al. 2013b, A\&A, 557, A105

Hada, K., Doi, A., Kino, M., et al. 2011, Nature, 477, 185

Kovalev, Y. Y., Lobanov, A. P., Pushkarev, A. B., \& Zensus, J. A. 2008, A\&A, 483, 759

Kutkin, A. M., Sokolovsky, K. V., Lisakov, M. M., et al. 2014, MNRAS, 437, 3396

Lister, M. L., Aller, H. D., Aller, M. F., et al. 2009, AJ, 137, 3718

Lobanov, A. P. 1998, A\&A, 330, 79

Marcaide, J. M., \& Shapiro, I. I. 1984, ApJ, 276, 56

Marscher, A. P. 2011, Nature, 477, 164

Marscher, A. P., Jorstad, S. G., Gómez, J.-L., et al. 2002, Nature, 417, 625

Marscher, A. P., Jorstad, S. G., D’ Arcangelo, F. D., et al. 2008, Nature, 452, 966

Marscher, A. P., Jorstad, S. G., Larionov, V. M., et al. 2010, ApJ, 710, L126

Molina, S. N., Agudo, I., Gómez, J. L., et al. 2014, A\&A, 566, A26

Perucho, M., Agudo, I., Gómez, J. L., et al. 2008, A\&A, 489, L29

Perucho, M., Kovalev, Y. Y., Lobanov, A. P., Hardee, P. E., \& Agudo, I. 2012, ApJ, 749, 55

Schmidt, M. 1965, ApJ, 141, 1295

Stanghellini, C., O’Dea, C. P., Dallacasa, D., et al. 1998, A\&AS, 131, 303

Zamaninasab, M., Savolainen, T., Clausen-Brown, E., et al. 2013, MNRAS, 436, 3341

Zamaninasab, M., Clausen-Brown, E., Savolainen, T., \& Tchekhovskoy, A. 2014, Nature, 510, 126

Zensus, J. A. 1997, ARA\&A, 35, 607 